\title{
NEURORETINITIS POR Bartonella henselae: A PROPÓSITO DE UN CASO CON SEGUIMIENTO POR TOMOGRAFÍA DE COHERENCIA ÓPTICA
}

\author{
Deivy Cruzado-Sánchez ${ }^{1, a}$, Camilo Tobón ${ }^{1, a}$, Vanesa Lujan ${ }^{1, a}$, Silvio Lujan ${ }^{1, a}$, Vanesa Valderrama 1,a
}

\section{RESUMEN}

Se reporta el caso de un varón de 36 años con neurorretinitis por Bartonella henselae, cuyo seguimiento periódico fue realizado con tomografía de coherencia óptica (TCO). El inicio de la enfermedad se caracterizó por disminución de agudeza visual (AV) unilateral, indolora, de inicio brusco en ojo derecho (OD), asociado a cuadro febril. El examen fundoscópico mostró edema en polo posterior, extendiéndose desde el disco óptico hasta la región macular en OD. La TCO confirmó el engrosamiento macular y del disco óptico así como la presencia de fluido macular subretinal. Los estudios sistémicos fueron normales con excepción del hemograma por presencia de leucocitosis y serología positiva a Bartonella henselae. El seguimiento tomográfico permitió valorar la disminución del edema macular, con la correspondiente mejora de la agudeza visual y ausencia de complicaciones asociadas. Este reporte describe la utilidad del seguimiento con TCO en un paciente con neurorretinitis por Bartonella henselae.

Palabras clave: Nervio óptico; Bartonella henselae; tomografía de coherencia óptica (fuente: DeCS BIREME).

\section{NEURORETINITIS CAUSED BY Bartonella henselae: A CASE WITH FOLLOW UP THROUGH OPTICAL COHERENCE TOMOGRAPHY}

\begin{abstract}
The case of a 36 year-old male with neuroretinitis caused by Bartonella henselae is reported, whose periodic follow-up was done through optical coherence tomography (OCT). The onset of this disease was characterized by unilateral low visual acuity (VA), painless, of sudden onset, in the right eye (RE), associated to I febrile symptom. The funduscopic examination showed edema in the posterior pole which extended from the optical disc to the macular region in the RE. The OCT confirmed macular and optical disc thickening, as well as the presence of subretinal macular fluid. Systemic studies were normal except for a blood count due to the presence of leukocytosis and positive for Bartonella henselae. The follow up with CT Scan helped to evaluate the decrease in macular edema, with the subsequent improvement of visual acuity and absence of related complications. This report describes the utility of the follow up with OCT in a patient with neuroretinitis caused by Bartonella henselae.
\end{abstract}

Key words: Optic nerve; Bartonella henselae; Tomography, optical coherence (source: MeSH NLM).

\section{INTRODUCCIÓN}

La infección por las especies de Bartonella es un problema de interés para la salud pública, de sus múltiples manifestaciones clínicas destaca la neurorretinitis, la cual es una entidad clínica caracterizada por la inflamación de la retina y del nervio óptico, asociada a múltiples causas siendo la forma infecciosa por bartonelosis la más frecuente ${ }^{(1)}$. El género Bartonella incluye más de 20 especies, estas se caracterizan por ser bacterias Gram negativas, intracelulares, y hemotrópicas ${ }^{(2)}$. Las especies B. henselae, B. quintana, B. grahamii y B. elizabethae son las que principalmente producen manifestaciones oculares, especialmente neurorretinitis ${ }^{(3)}$.

La tomografía de coherencia óptica (TCO) es un método no invasivo y reproducible que brinda imágenes de alta resolución de las capas de la retina; con el tiempo se ha constituido en una ayuda diagnóstica importante para el oftalmólogo pues permite valorar con alta precisión la evolución de las lesiones que se producen en la mácula y el nervio óptico ${ }^{(4)}$. Se ha descrito el uso de la TCO en neurorretinitis en algunas series de casos ${ }^{(5)}$; sin embargo, las imágenes son de menor resolución denominadas de

\footnotetext{
Centro Oftalmológico Mácula D\&T. Lima, Perú.

Médico oftalmólogo
}

Recibido: 05-10-12 Aprobado: 23-01-13

Citar como: Cruzado-Sánchez D, Tobón C, Lujan V, Lujan S, Valderrama V. Neuroretinitis por Bartonella henselae: a propósito de un caso con seguimiento por tomografía de coherencia óptica. Rev Peru Med Exp Salud Publica. 2013;30(1):133-6. 
dominio de tiempo. El objetivo de este reporte de caso es describir el seguimiento tomográfico de un caso con neurorretinitis por Bartonella henselae.

\section{REPORTE DE CASO}

Varón de 36 años que desde hace un día acusa disminución de agudeza visual (AV) unilateral, indolora y de inicio brusco en ojo derecho (OD) similar a una "mancha oscura central". Asimismo, refiere que cinco días previos a este evento cursó con cefalea leve y cuadro febril que cedió con acetaminofén vía oral, sin complicación alguna. Otro dato de referencia fue el contacto cotidiano con un gato doméstico.

Al examen oftalmológico se encontró agudeza visual corregida de 20/160 en ojo derecho (OD) y 20/20 en ojo izquierdo $(\mathrm{OI})$, además de defecto pupilar aferente relativo en OD. En el campo visual por confrontación se evidenció un escotoma central en ese mismo ojo. Por biomicroscopía se apreció leve hiperemia ciliar, y en el segmento anterior no se observó efecto Tyndall.
El examen fundoscópico mostró edema de disco óptico unilateral y edema difuso retinal que se extienden desde el disco hasta la región macular (Figura 1).

Los diagnósticos diferenciales incluyeron neurorretinitis, oclusiones vasculares isquémicas y papiledema unilateral. Para ello se realizaron diversos exámenes auxiliares, como hemograma, glucosa, velocidad de sedimentación globular (VSG), Elisa para VIH, inmunoglobulinas para toxoplasmosis, ANA, ANCA, factor reumatoide, VDRL, resonancia magnética nuclear cerebral con y sin contraste y PPD. Todos fueron normales con excepción de la presencia de leucocitosis en el hemograma de $18000 / \mathrm{mm}^{3}$ leucocitos, y serología positiva para Bartonella por el método de Elisa con títulos de lg G: 45,0 e lg M: 10,9.

La tomografía de coherencia óptica (TCO) confirmó el edema de la mácula y del disco óptico, así como la presencia de fluido macular subretinal (Figura 2). El examen de campimetría computarizada confirmó el escotoma central en el OD. El examen angiográfico con fluoresceina aportó la fuga del colorante a nivel de la

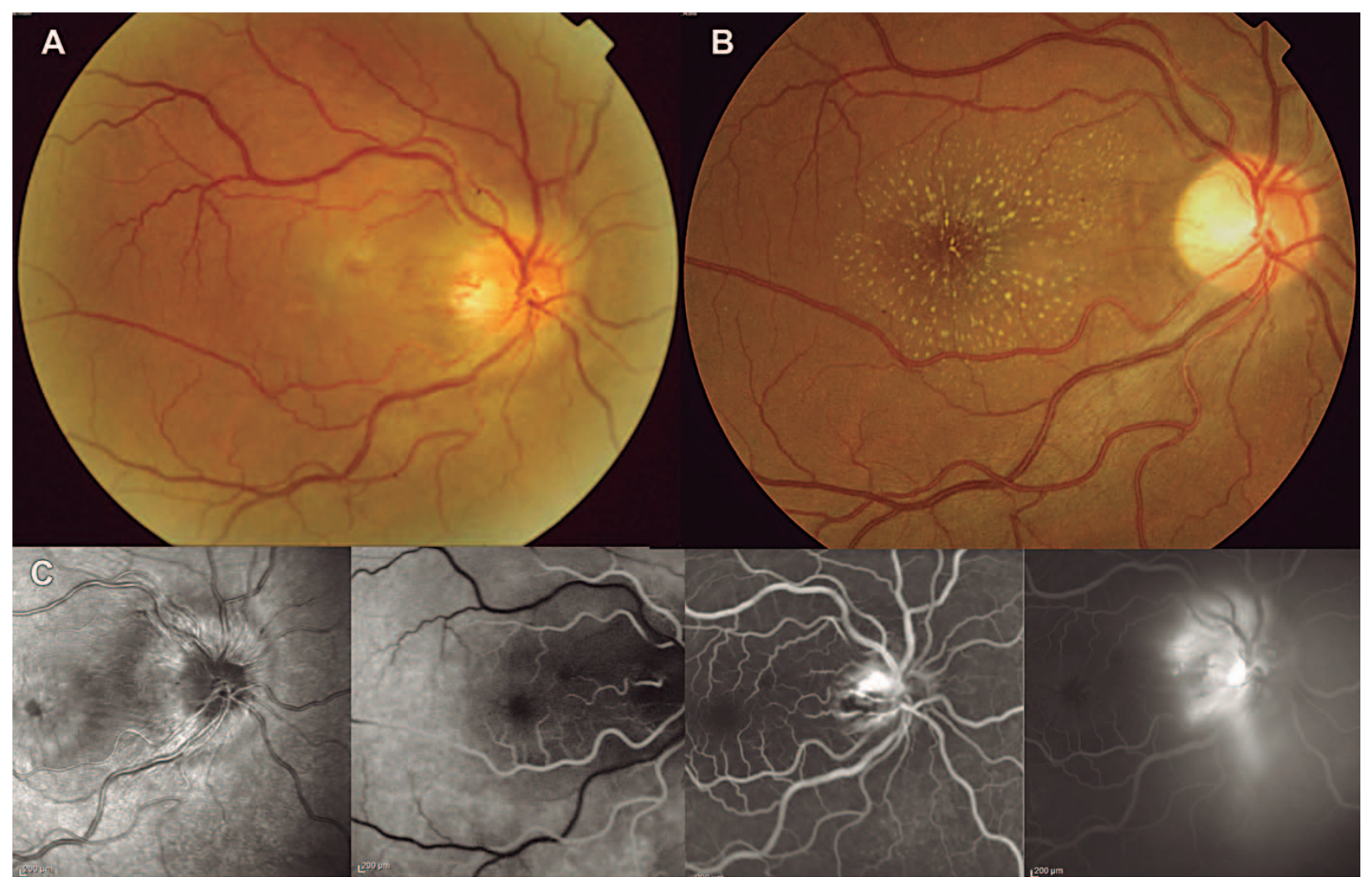

Figura 1. Retinografías a color y angiografía con fluoresceína.

A) Retinografía a color derecha, muestra el día 1, y se observa el edema a nivel del nervio óptico. B) A los 10 días posteriores se puede observar en la retinografía de la izquierda los depósitos lipídicos ordenados radialmente característicos de la estrella macular. C) En el angiograma se puede observar la fuga de colorante a nivel del nervio óptico en tiempo tardío. 




Figura 2. Seguimiento tomográfico a nivel macular y agudeza visual.

Se observa la presencia de fluido subretinal y el engrosamiento de las capas externas de la retina principalmente de los fotorreceptores y plexiforme. Asimismo, se observan los cambios favorables en la agudeza visual del correlacionándose con la arquitectura histológica del paciente.

cabeza del nervio óptico en los tiempos tardíos, pero con escasos cambios en la mácula (Figura 1)

Se diagnosticó neurorretinitis por Bartonella henselae. El paciente fue tratado con ciprofloxacino $500 \mathrm{mg}$ bid por 3 semanas y prednisona $75 \mathrm{mg}$ por día por una semana, ambos por vía oral; además, se le indicó acetato de prednisolona al $1 \%$ y ciprofloxacino $0,3 \%$ por vía tópica ocular.

Durante el seguimiento, se evidencia a la segunda semana, en la retinografía a color, el signo de la "estrella macular" que caracteriza a esta enfermedad (Figura 1). La TCO mostró los cambios favorables caracterizados por disminución del edema de la mácula y el nervio óptico con la consecuente mejora de la agudeza visual de $20 / 25$ en OD. Se siguió este caso con controles tomográficos seriados hasta el día 90 desde su ingreso (Figura 2 y 3 ).

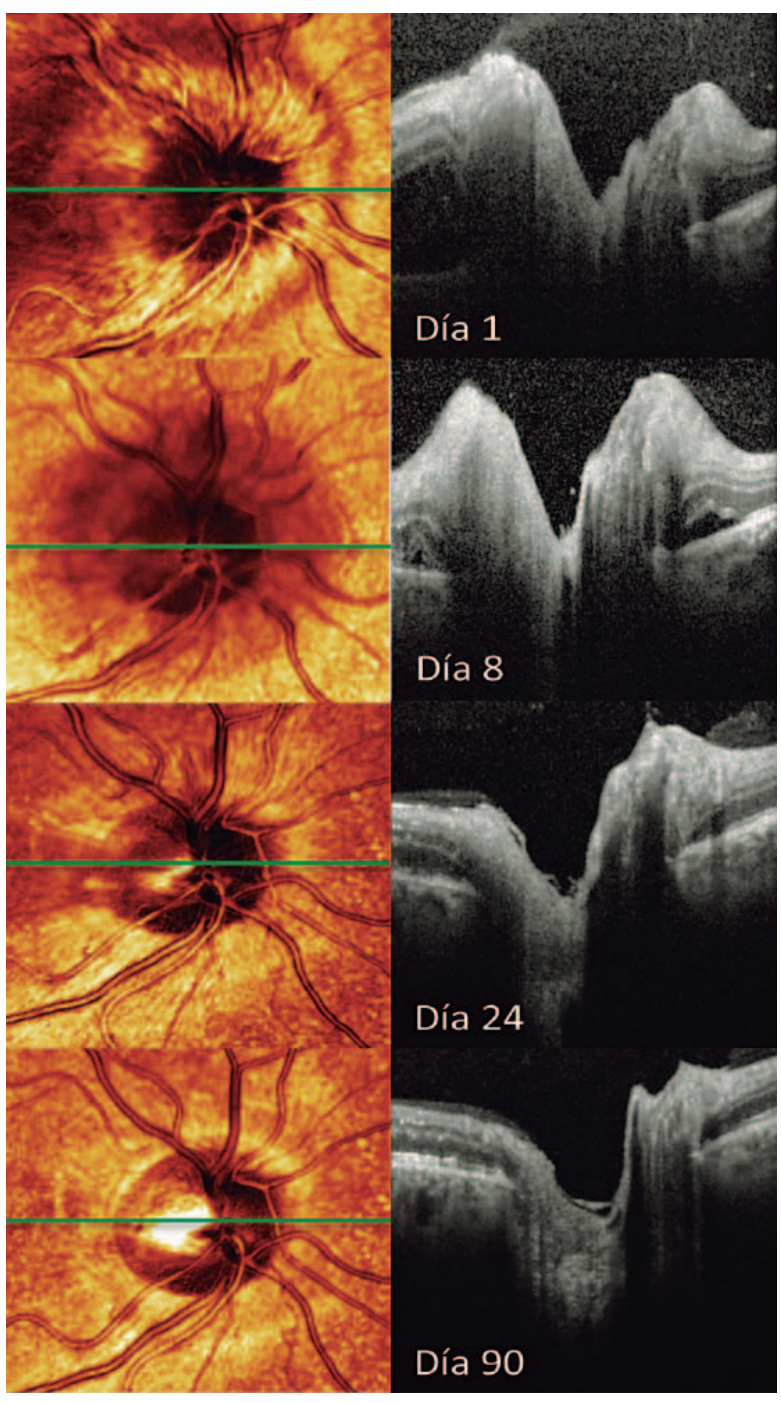

Figura 3. Seguimiento tomográfico a nivel del nervio.

Se puede observar cómo el engrosamiento de las fibras nerviosas del nervio óptico van disminuyendo con el tiempo, así como la desaparición del fluido subretinal asociado.

\section{DISCUSIÓN}

La bartonelosis es un cuadro sistémico variado, cuyos signos y síntomas incluyen cefalea, fatiga, fiebre y adenopatías que, generalmente se autolimitan ${ }^{(3)}$. En este caso el paciente presentó cinco días previos esta sintomatología, pero sin la presencia de linfadenopatías, ni lesiones cutáneas.

El diagnóstico se basó en la clínica, antecedentes, exámenes auxiliares y la serología positiva para Bartonella. Sin embargo, se ha reportado limitaciones en la sensibilidad de esta prueba y su discriminación con otras especies de Bartonella ${ }^{(6,7)}$, otras técnicas diagnósticas de mayor sensibilidad como la reacción en cadena de polimerasa podrían haber permitido definir la especie de Bartonella. 
El uso de la TCO permitió describir la mácula pues se observó las alteraciones en la capas de la retina, principalmente el engrosamiento en las capas externas de la retina como la plexiforme y los fotorreceptores; además de la presencia de fluido subretinal a nivel foveal, y de puntos hiperreflectivos que se correlacionan con depósitos del material lipídico a nivel intrarretinal, que posteriormente toman una disposición radial configurando la estrella macular que caracteriza a esta enfermedad (Figura 1).

Durante el seguimiento tomográfico se puede observar la disminución del grosor retinal de $1150 \mu \mathrm{m}$ a $256 \mu \mathrm{m}$ a expensas de la ausencia del fluido subretinal y la disminución del engrosamiento de las capas externas de la retina (fotorreceptores y plexiforme); asimismo, es notoria la reabsorción lenta del material lipídico depositado. Estas mismas características ya habían sido descritas por Habot-Wilmer $Z$ et al., sin embargo, sus imágenes se sustentaban en TCO de menor resolución denominadas de dominio de tiempo ${ }^{(5)}$, donde el corte tomográfico presentado está realizado a razón de 400 barridos por segundo, comparado con el empleado en este estudio, de 40000 barridos denominado TCO de dominio espectral, con el que se obtienen mejoras cualitativas en la calidad de las imágenes ${ }^{(8)}$.
Los cortes tomográficos en el nervio óptico muestran el engrosamiento de las fibras nerviosas, HabotWilmer Z. et al. ${ }^{(5)}$ no habían reportado estos hallazgos por la naturaleza retrospectiva de sus estudios. En este estudio se evidencia en el seguimiento, cómo el proceso inflamatorio de la capa de fibras nerviosas va disminuyendo en el tiempo. Durante el seguimiento no se presentaron complicaciones asociadas tales como obstrucción vascular o desprendimiento de retina.

En conclusión, el reporte de este caso valora la utilidad de la tomografía de coherencia óptica para el seguimiento de un caso de neurorretinitis por bartonelosis, permitiéndonos cuantificar los cambios histológicos producidos en las capas de la retina y a nivel del nervio óptico, y poder estar al tanto de las posibles complicaciones que se puedan presentar.

Contribuciones de autoría: DCS y CTM participaron en la concepción del artículo y recolección de los datos. Todos los autores participaron de la interpretación de los datos, redacción del artículo, revisión crítica y aprobación final.

Fuentes de financiamiento: autofinanciado.

Conflictos de interés: los autores declaran no tener ningún conflicto de interés.

\section{REFERENCIAS BIBLIOGRÁFICAS}

1. Reed JB, Scales DK, Wong MT, Lattuada CP Jr, Dolan MJ, Schwab IR. Bartonella henselae neuroretinitis in cat scratch disease. Diagnosis, management, and sequelae. Ophthalmology. 1998;105(3):459-66.

2. Chomel BB, Boulouis HJ, Breitschwerdt EB, Kasten RW, Vayssier-Taussat M, Birtles RJ, et al. Ecological fitness and strategies of adaptation of Bartonella species to their hosts and vectors. Vet Res. 2009;40(2):29.

3. Kalogeropoulos C, Koumpoulis I, Mentis A, Pappa C, Zafeiropoulos P, Aspiotis M. Bartonella and intraocular inflammation: a series of cases and review of literature. Clin Ophthalmol. 2011;5:817-29.
4. Pinilla I, Ruiz-Moreno JM, Cuenca N. Correlación de la tomografía de coherencia óptica con la histología retiniana. Arch Soc Esp Oftalmol. 2012;87(9):275-7.

5. Habot-Wilner Z, Zur D, Goldstein M, Goldenberg D, Shulman S, Kesler A, Giladi M, Neudorfer M. Macular findings on optical coherence tomography in cat-scratch disease neuroretinitis. Eye (Lond). 2011;25(8):1064-8.

6. Bergmans AMC, Peeters MF, Schellekens JFP, Vos MC, Saabe LJM, Ossewaarde $\mathrm{JM}$, et al. Pitfalls and fallacies of cat scratch disease serology: evaluation of Bartonella henseleae-based indirect fluorencence assay and enzyme-linked immunoassay. J Clin Microbiol. 1997;35(8):1931-7
7. Metzkor-Cotter E, Kletter Y, Avidor B, Varon M, Golan Y, Ephros M, et al. Long term serological analysis and clinical follow-up of patients with cat scratch disease. Clin Infect Dis. 2003;37(9):1149-54.

8. Grover S, Murthy RK, Brar VS, Chalam $\mathrm{KV}$. Comparison of retinal thickness in normal eyes using Stratus and Spectralis optical coherence tomography. Invest Ophthalmol Vis Sci. 2010;51(5):2644-7.

Correspondencia: Deivy Cruzado Sánchez. Dirección: Carlos Ferreyros 120. Oficina 301-401. Lima 27, Perú.

Teléfono: (511) 226-3801

Correo electrónico:dcruzados@gmail.com 